\title{
Cross-sectional study of asymptomatic malaria and seroepidemiological surveillance of seven districts in Gia Lai province, Vietnam
}

Nguyen Ngoc San ${ }^{1 \dagger}$, Nguyen Xuan Kien ${ }^{2+}$, Nguyen Duc Manh ${ }^{3}$, Nguyen Van Thanh ${ }^{3}$, Marina Chavchich4, Nguyen Thi Huong Binh ${ }^{5}$, Tran Khanh Long ${ }^{6}$, Kimberly A. Edgel ${ }^{7}$, Eduard Rovira-Vallbona ${ }^{6 *}$, Michael D. Edstein ${ }^{4}$ and Nicholas J. Martin ${ }^{7}$

\begin{abstract}
Background: Malaria elimination by 2030 is an aim of many countries in the Greater Mekong Sub-region, including Vietnam. However, to achieve this goal and accelerate towards malaria elimination, countries need to determine the extent and prevalence of asymptomatic malaria as a potential reservoir for malaria transmission and the intensity of malaria transmission. The purpose of this study was to determine the prevalence of asymptomatic malaria and seropositivity rate in several districts of Gia Lai province in the Central Highlands of Vietnam.
\end{abstract}

Methods: A cross-sectional survey of asymptomatic malaria and serological testing was conducted in 3283 people living at 14 communes across seven districts in Gia Lai province in December 2016 to January 2017. Finger prick capillary blood samples were tested for malaria using rapid diagnostic testing and polymerase chain reaction (PCR), as well as detecting antibodies against 3 Plasmodium falciparum and 4 Plasmodium vivax antigens by indirect enzyme-linked immunosorbent assay (ELISA). Age-seroprevalence curves were fitted using reverse catalytic models with maximum likelihood.

Results: The study population was predominantly male $(65.9 \%, 2165 / 3283)$, adults $(88.7 \%, 2911 / 3283)$ and of a minority ethnicity $(72.2 \%, 2371 / 3283)$, with most participants being farmers and outdoor government workers $(90.2 \%$, 2960/3283). Using a small volume of blood $(\approx 10 \mu \mathrm{L})$ the PCR assay revealed that $1.74 \%$ (57/3283) of the participants had asymptomatic malaria (P. falciparum 1.07\%, P. vivax 0.40\%, Plasmodium malariae $0.15 \%$ and mixed infections $0.12 \%)$. In contrast, the annual malaria prevalence rates for clinical malaria in the communities where the participants lived were $0.12 \%(108 / 90,395)$ in 2016 and $0.22 \%(201 / 93,184)$ in 2017 . Seropositivity for at least one P. falciparum or one P. vivax antigen was 38.5\% (1257/3262) and 31.1\% (1022/3282), respectively. Age-dependent trends in the proportion of seropositive individuals in five of the districts discriminated the three districts with sustained low malaria prevalence from the two districts with higher transmission.

Conclusions: Asymptomatic Plasmodium carriers were found to be substantially more prevalent than clinical cases in seven districts of Gia Lai province, and a third of the population had serological evidence of previous malaria

\footnotetext{
*Correspondence: edu.rovira@outlook.com

${ }^{\dagger}$ Nguyen Ngoc San and Nguyen Xuan Kien contributed equally to this

work

${ }^{6}$ Vysnova Partners, Bethesda, MD, USA

Full list of author information is available at the end of the article
}

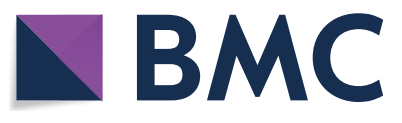

Ther(s) 2022. Open Access This article is licensed under a Creative Commons Attribution 4.0 International License, which permits use, sharing, adaptation, distribution and reproduction in any medium or format, as long as you give appropriate credit to the original author(s) and the source, provide a link to the Creative Commons licence, and indicate if changes were made. The images or other third party material in this article are included in the article's Creative Commons licence, unless indicated otherwise in a credit line to the material. If material is not included in the article's Creative Commons licence and your intended use is not permitted by statutory regulation or exceeds the permitted use, you will need to obtain permission directly from the copyright holder. To view a copy of this licence, visit http://creativecommons.org/licenses/by/4.0/. The Creative Commons Public Domain Dedication waiver (http://creativeco mmons.org/publicdomain/zero/1.0/) applies to the data made available in this article, unless otherwise stated in a credit line to the data. 
exposure. The findings add knowledge on the extent of asymptomatic malaria and transmission for developing malaria elimination strategies for Vietnam.

Keywords: Asymptomatic malaria, Plasmodium falciparum, Plasmodium vivax, Gia Lai province, Vietnam, PCR, Serology, Drug resistance

\section{Background}

Despite continued decreases in the number of malaria cases outside of Africa, there are still more than 200,000 cases reported annually across Asia and the Pacific, primarily in 11 endemic countries (Cambodia, China, the Lao People's Democratic Republic, Myanmar, Malaysia, Papua New Guinea, the Philippines, the Republic of Korea, Solomon Islands, Vanuatu and Vietnam) [1]. Countries across the Greater Mekong Subregion (GMS) have pledged to eliminate all Plasmodium species of malaria by 2030 [2]. There is lasting value to eliminating malaria as nearly all countries that have achieved malaria elimination have been able to maintain this status despite the presence of competent vectors, imported cases and proximity to malaria endemic countries [3]. Additionally, malaria elimination is critical to combating drug resistant malaria present across the GMS [4].

Significant reductions in malaria mortality and morbidity were observed in Vietnam between 1991 and 2013 with continued decreases reported through 2015 [5]. However, the Vietnam National Institute of Malariology, Parasitology and Entomology (NIMPE) reported an increase in the overall number of malaria cases during 2017-2018. Challenges complicating elimination plans are: (1) the presence of $P$. falciparum parasites resistant to the current artemisinin-based first-line treatments described in six provinces (Quang Nam, Gia Lai, Dak Nong, Khanh Hòa, Ninh Thuan and Binh Phuoc) [4], (2) identification and targeting of the sub-clinical infectious reservoir, and (3) the elimination of infections caused by malaria species other than $P$. falciparum. While P. falciparum is the focus of current elimination efforts, malaria caused by Plasmodium vivax, Plasmodium malariae, Plasmodium ovale and Plasmodium knowlesi may require different strategies due to either dormant liver stages (hypnozoites), importation from areas outside of Vietnam or presence of animal reservoirs [5-7].

It is widely accepted that asymptomatic malaria parasite carriers present a unique challenge for elimination programmes as they provide a transmission reservoir capable of sustaining malaria endemicity [8-10]. As Vietnam and other countries approach zero cases of malaria, a pre-requisite for World Health Organization (WHO) designation of malaria elimination, areas of residual transmission will become increasingly important to identify and treat. National Malaria Control Programmes
(NMCPs) still rely almost exclusively on blood film microscopy or rapid diagnostic tests (RDTs) for the routine detection of malaria and case investigations at communal health stations and for large scale malaria surveys. Although these tools are relatively inexpensive and widely available, they lack the sensitivity to detect infections in individuals who are asymptomatic and/or carry sub-microscopic parasite densities. These infections need to be identified by more sensitive molecular tools, such as polymerase chain reaction (PCR), to aid NMCPs develop elimination strategies and target resources to areas of residual malaria transmission.

As transmission decreases, parasitological surveys designed to identify ongoing infections will require large sample sizes, becoming logistically and economically unfeasible for NMCPs. Other malaria transmission metrics such as entomological inoculation rate also have inherent limitations in low transmission areas due to very low numbers of infected mosquitoes [11-13]. Alternatively, serological markers of parasite exposure have been proposed as a potentially cost-effective proxy for malaria transmission intensity in low endemic areas $[10$, $11,14,15]$. Seropositivity to parasite antigens in a specific population is assumed to represent cumulative exposure to past malaria infections thus reducing the probability of missing exposure events, including asymptomatic infections. Moreover, by analysing age-seroprevalence patterns it is possible to identify historical trends in transmission intensity in a specific population group and how these trends differ between communities [11, 16, 17]. Such serological tools are of special interest in preelimination scenarios existing in Vietnam, with marked spatial transmission heterogeneity (i.e. hotspots of transmission surrounded by areas of no transmission), where geographical stratification in transmission levels becomes essential to target NMCP interventions efficiently [18].

The primary objective of this cross-sectional study was to characterize the parasite reservoir of $P$. falciparum, $P$. vivax, $P$. ovale, $P$. malariae and $P$. knowlesi in an asymptomatic population working and residing in Gia Lai province, Vietnam. Three endpoints were assessed to characterize malaria burden in the study population: (1) prevalence of parasite positive individuals determined by PCR, (2) prevalence of individuals with a history of malaria exposure measured by detection of antibodies to $P$. falciparum or $P$. vivax, and (3) proportion of $P$. 
falciparum parasites with molecular markers associated with drug resistance.

\section{Methods \\ Study location}

The study was conducted between December 2016 and January 2017 at located in seven different districts of Gia Lai province. Gia Lai is located in the Central Highlands of Vietnam on the border with Cambodia, has a population of approximately 1.3 million people residing in an area of $15,500 \mathrm{~km}^{2}$ distributed in 17 districts and city divisions (Fig. 1). Malaria transmission is perennial, typically rising after the start of the rains in May and peaking between September and December, with the lowest incidence from February to April. Delayed P. falciparum malaria clearance following artemisinin-based combination therapy (i.e. dihydroartemisinin-piperaquine, DHAPPQ) was reported in Gia Lai province in 2010 and 2017 $[19,20]$.

Cross-sectional malaria surveys were done in 14 military-civilian health facilities across the seven districts: Chu Prong (Ia Mo and Ia Puch communes), Chu Puh (Ia Hla, Ia Le and Ia Phang communes), Chu Se (Ia Hlop and Ia Ko communes), Duc Co (Ia Dom, Ia Nan and Ia Pnon communes), Ia Grai (Ia Chia and Ia O communes), Kong Chro (So Ro commune), and Krong Pa (Chu RCam commune) (Fig. 1). Subjects residing and/or working in these areas include both military personnel and civilians, who regularly receive primary health care at military medical facilities. Chu Pong, Duc Co and Ia Grai districts are located close to the border with Cambodia, where individuals are mainly responsible of border security (Fig. 1). Chu Se and Chu Puh are located in the centre of the province, with a predominance of soldiers working in farming. Finally, Kong Chro and Krong Pa districts are in the east, with predominance of local civilian population.

\section{Sample size}

The asymptomatic malaria prevalence was assumed to be greater than $1.25 \%$ based on a malaria survey conducted in Krong Pa district in April 2016, in which three malaria cases were detected by blood film microscopy out of 240 people screened (Dr. Ro Mah Huan, Center for Disease Control and Prevention, Gia Lai province, unpublished data). It was determined that a minimum of 3,052 participants would be required to detect a PCR positive rate of $\geq 1.25 \%$ with a two-sided $95 \%$ confidence interval no wider than $1 \%$ with alpha and beta errors of 0.05 and 0.20 , respectively. Allowing for $7 \%$ to $8 \%$ of subjects withdrawing their consent to participate in the study, it was decided to recruit at least 3,265 to 3,296 participants.

\section{Volunteer recruitment and enrolment}

Both asymptomatic civilians and government workers from the Vietnam People's Army eligible for medical care in the area covered by the selected military medical facilities were non-randomly invited to participate in the study. The inclusion criteria for the study were: (1) working or residing in Gia Lai province during the 14 days prior to the study visit, (2) 5 to 65 years of age, and (3) willingness to provide information (e.g. occupation, recent travel history, health check) and a finger prick blood sample. The exclusion criteria were: (1) being unwilling to provide consent, information or finger prick capillary blood sample, (2) inability to communicate well with the study staff, and (3) presenting with tympanic body temperature $>38{ }^{\circ} \mathrm{C}$ or reporting other malaria symptoms such as headache; these individuals were referred to the nearest health care facility. Following informed consent, study staff collected finger prick capillary blood samples for RDT (SD Bioline Malaria Ag Pf/Pv, Standard Diagnostics Inc., Abbott, Princeton, NJ, USA) and four filter paper blood spot samples were collected on 3MM filter paper (Whatman, Buckinghamshire, UK) for PCR analysis and antibody detection. Blood spots were stored individually in zip-closure plastic bags with desiccant (10 silica gel beads). Body temperature was measured, and demographic information was collected.

\section{Detection of malaria by PCR}

DNA was extracted from filter paper blood spots (approximately $10 \mu \mathrm{L}$ of whole blood) using the QIAamp DNA mini kit (Qiagen, Hilden, Germany) as per manufacturer's protocol and eluted in $150 \mu \mathrm{L}$ of AE buffer. Five $\mu \mathrm{L}$ was used as template in a single round multiplex PCR targeting small subunit (SSU) ribosomal RNA for simultaneous detection of $P$. falciparum, $P$. vivax, $P$. ovale, and P. malariae, as previously described [21]. PCR products were analysed by gel electrophoresis on $3 \%$ Tris-Acetate EDTA gel and compared to those produced by positive controls. Sensitivity of this method for detection of $P$. falciparum was tested using standards prepared from P. falciparum D6 laboratory line (Sierra-Leone, Africa). Briefly, highly synchronous ring-stage parasite cultures at $5 \%$ parasitaemia were centrifuged, washed twice in 100\% plasma and resuspended at $50 \%$ haematocrit. These suspensions were dispensed into $50 \mu \mathrm{L}$ aliquots in LoBind Eppendorf tubes and stored at $-80^{\circ} \mathrm{C}$. The parasite DNA was extracted from $50 \mu \mathrm{L}$ aliquots and serially diluted. Using these standards, the lower limit of detection (LOD) for PCR was $\geq 0.25$ parasites $/ \mu \mathrm{L}$. The PCR performance acceptance threshold was set tenfold greater than LOD at $\geq 2.5$ parasites $/ \mu \mathrm{L}$ (equivalent to 4.16 parasites in a 


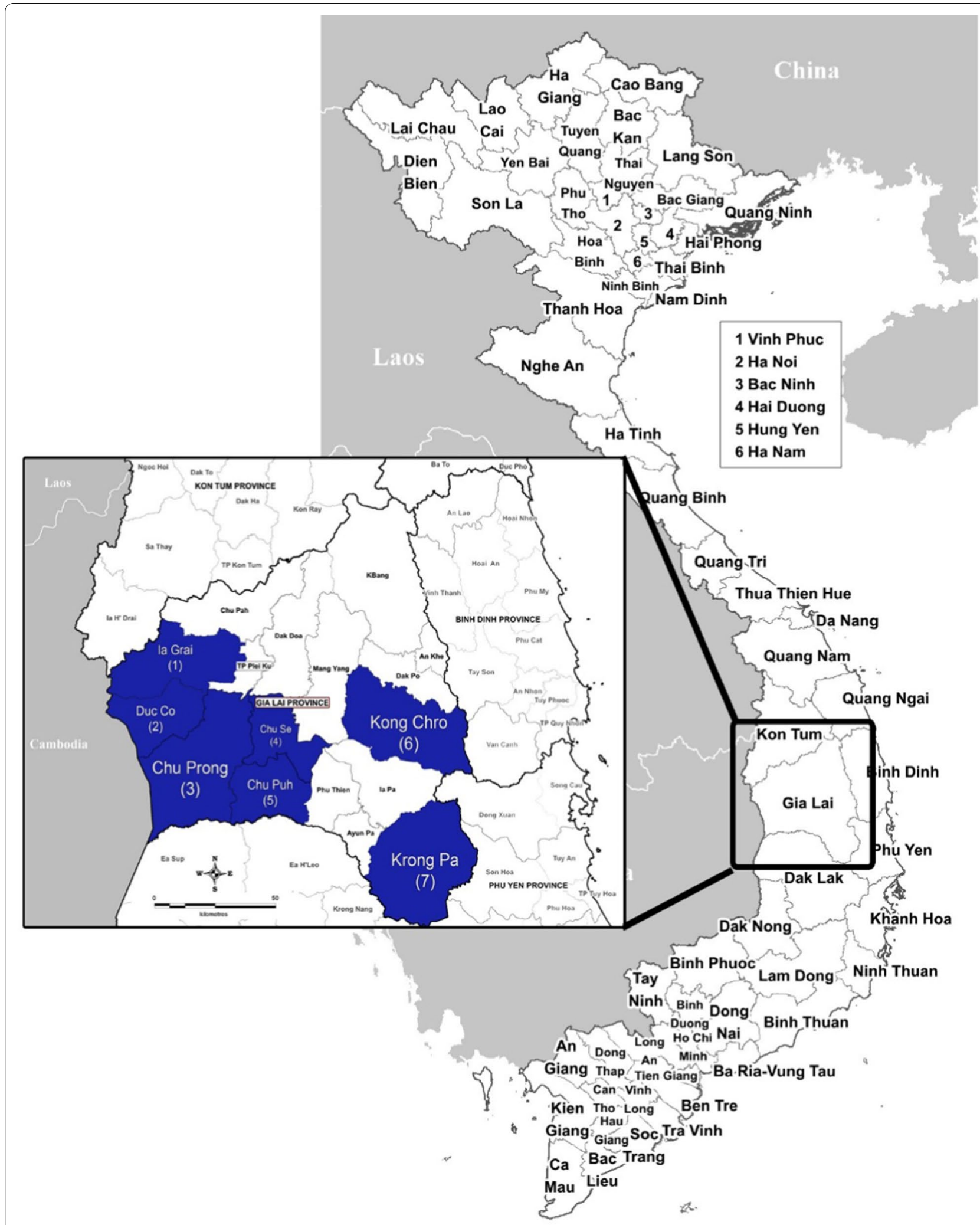

Fig. 1 Map of Gia Lai province, Vietnam. The study districts highlighted are in blue: la Grai (1), Duc Co (2), Chu Prong (3), Chu Se (4), Chu Puh (5), Kon Chro (6) and Krong Pa (7) 
PCR reaction or 0.833 parasites/ $\mu \mathrm{L}$ DNA eluate) with respective positive DNA controls included into every batch of PCR analysis. As DNA extraction efficiency control, filter paper spots containing 50 parasites per spot were prepared using the synchronous ring-stage D6 parasites and included in every batch of DNA extraction and subsequent PCR. When testing field samples, positive PCR results were accepted (i.e. bands on the gel of the size corresponding to the respective positive controls) if they were reproduced in at least 2 out of 3 PCR conducted. In addition, detection of $P$. knowlesi was carried out as previously described by Imwong et al. [22].

\section{Molecular markers of drug resistance}

Prevalence of artemisinin-resistance validated mutations in the P. falciparum kelch-13 gene (WHO report on artemisinin and ACT resistance, August 2018, [23]; i.e. F446I, N458Y, M476I, Y493H, R539T, I543T, P553L, R561H and $\mathrm{C} 580 \mathrm{Y}$ ) were evaluated by sequencing of the $850 \mathrm{bp}$ Pfkelch-13 gene region covering amino acid 427 to 687, using a previously described method [24]. For markers of piperaquine resistance, mutation in exo-E415G (exonuclease gene PF3D7_1362500) and copy number of plasmepsin 2 and 3 were analysed as previously described $[25,26]$. In addition, mutations in the $P$. falciparum chloroquine resistance transporter $(P f c r t)$ gene at codons 72 to 76 associated with chloroquine resistance were analysed by PCR amplification and sequencing, as previously described [27]. Copy number of $P$. falciparum multidrug resistance protein 1 (Pfmdr1) gene, which is associated with lumefantrine and mefloquine resistance, was determined by qPCR using SYBR Green chemistry [28]. Copy numbers $>1.5$ was considered a gene duplication.

\section{Serological tests}

Antibodies against $P$. falciparum and $P$. vivax were detected by indirect enzyme-linked immunosorbent assay (ELISA). For antibody elution, two $5 \mathrm{~mm}$ discs were punched from dried blood spots, placed in a 96-well plate, and mixed with $1,120 \mu \mathrm{L}$ of elution buffer containing PBS/0.05\% Tween20/0.05\% $\mathrm{NaN}_{3}$. Plates were incubated overnight at ambient temperature $\left(\sim 23{ }^{\circ} \mathrm{C}\right)$ and with shaking $(200 \mathrm{rpm})$, and pink/red colored eluates were stored at $4{ }^{\circ} \mathrm{C}$. The following recombinant proteins were produced: PfAMA-1 (3D7 strain), PfMSP1 (19 kD C-terminal fragment, 3D7), PfCSP (3D7), PvAMA-1 (Palo Alto), PvMSP1 (19 kD C-terminal fragment), PvCSP allelic variant 210 (Sal-1) and PvCSP allelic variant 247 (PNG_M69059.1). All proteins were produced at Burnet Institute (Melbourne, Australia), except PfMSP1 and PvMSP1 produced at Dalian Institute of Biotechnology (Dalian, China).
ELISA was then used to measure IgG recognition of recombinant proteins. Briefly, 96-well microplates (Corning, Corning, NY, USA) were coated with $25 \mathrm{ng} /$ well of antigen in PBS or carbonate buffer, sealed and incubated overnight at $4{ }^{\circ} \mathrm{C}$. Total IgG from human serum (Sigma-Aldrich, St. Louis, MS, USA) were used as plate standards and directly coated in control wells at three different dilutions (50 ng, $25 \mathrm{ng}$ and $12.5 \mathrm{ng}$ ). Plates were washed and blocked with $1 \%$ skimmed milk powder in PBS $/ 0.05 \%$ Tween 20 for $2 \mathrm{~h}$. Fifty $\mu \mathrm{L}$ of antibody eluates were tested in duplicate at $1 / 200$ dilution together with positive (confirmed malaria infected patients by light microscopy) and a negative control (unexposed Vietnamese). After incubation at room temperature for $2 \mathrm{~h}$, peroxidase-conjugated goat anti-human IgG secondary antibody (Sigma-Aldrich) was added for $1 \mathrm{~h}$, washed and mixed with $50 \mu \mathrm{L}$ of 2,2'-azino-di-(3-ethylbenzthiazoline sulfonic acid (Sigma-Aldrich). Reaction was stopped after 15 min with $50 \mu \mathrm{L}$ of $2 \mathrm{M} \mathrm{H}_{2} \mathrm{SO}_{4}$, and optical density (OD) values read at $405 \mathrm{~nm}$ in a Multiskan ${ }^{\mathrm{TM}}$ FC Microplate Photometer (ThermoFisher, Waltham, MA, USA). Duplicates with $>25 \%$ variation were repeated once, and those with $>25 \%$ variation after repetition were excluded from analysis. The mean OD from blank wells was subtracted from samples, controls and IgG standards at each plate, and total IgG standards were then used to normalize mean sample OD between plates. Fifteen percent of the study samples $(n=575)$ were retested for all seven antigens to assess reproducibility of serological assays, resulting in pairwise Pearson's correlation coefficients ranging $0.877-0.952$ (all $<5 \%$ Bonferroni-adjusted significance level, Additional file 1: Figure S1). The cut-off for ELISA seropositivity against each antigen was defined as the mean OD of negative controls plus three times the standard deviation.

\section{Definitions and statistical analysis}

The main dependent variable used this study was malaria infection based on PCR analysis. Independent demographic variables included age (stratified in seven age groups), ethnicity (Kinh versus ethnic minorities), sex, occupation (farmer, student, and government worker) and district.

Individuals were considered exposed to $P$. falciparum or $P$. vivax if they tested seropositive for at least one of the species antigens. Age-seroprevalence curves were fitted using reverse catalytic conversion models with maximum likelihood methods in R ( $\mathrm{R}$ Core Team, v3.5.1), as previously described [17]. The model calculates one seroconversion rate $(\lambda$, i.e. the rate at which seronegative individuals become seropositive per year) and one seroreversion rate $(\rho$, i.e. rate at which seropositive individuals become seronegative per year). Seroconversion rate can 
thus be used as a measure of malaria force of infection or transmission intensity, whereas seroreversion rates can be interpreted as a measure of persistence of the antibody response [11]. Differences in demographic characteristics and malaria prevalence by PCR or serological data were determined using Pearson's chi-squared $\left(\chi^{2}\right)$ tests with Bonferroni corrections, or Fisher's exact test when sample size was small. Risk factor analysis for $P$. falciparum and $P$. vivax seropositivity was conducted by running univariate logistic regression models for age, sex, ethnic group, occupation, district of residence and PCR positivity. Statistical analyses were performed in IBM SPSS 22.0 or Stata Version 12.0 (StataCorp, TX, USA), and graphs were created in Prism v8.0 (GraphPad, CA, USA). A significance level of $p<0.05$ was defined for all tests.

\section{Results}

\section{Characteristics of the study population}

A total of 3,283 individuals consented to participate and were enrolled in the study from health facilities in Chu Prong $(n=330)$, Chu Puh $(n=600)$, Chu Se $(\mathrm{n}=400)$, Duc Co $\mathrm{n}=530)$, Ia Grai $(\mathrm{n}=220)$, Kong Chro $(n=600)$ and Krong Pa $(n=603)$ districts. Overall, the participants were predominantly adults
( $\geq 18$ years of age; $88.7 \%, 2911 / 3283$ ), male (65.9\%, $2165 / 3283)$, and belonging to an ethnic minority group (72.2\%, 2371/3283), with most participants engaged in farming and outdoor work activities $(90.2 \%, 2960 / 3283)$ (Table 1). However, demographic characteristics between districts were heterogeneous: participants enrolled in Chu Puh and Chu Se districts were exclusively adult male government workers engaged in production labor in rubber and coffee farms $(n=1000)$, whereas participants from Kong Chro and Krong Pa were almost exclusively from local ethnic minority groups with a high proportion of individuals under 20 years of age $(\geq 30.9 \%$; other districts: $\leq 22.3 \%$ ).

The annual number of clinical malaria cases reported by the 14 communal health stations by microscopy and/or RDT between 2014 and 2017 are presented in Table 2 (detailed data for each district are presented in the Additional file 1: Table S1). Malaria prevalence (all species) declined from $0.77 \%(626 / 81,346)$ in 2014 to $0.12 \%(108 / 90,395)$ in 2016 , but increased to $0.22 \%$ $(201 / 93,184)$ in 2017 [29]. During this period, the average proportion of cases attributable to $P$. falciparum was $58.8 \%$ (range $38.9-73.6 \%$ ), whereas $P$. vivax accounted for $39.7 \%(24.9-59.2 \%)$.

Table 1 Study population demographic characteristics at the seven districts in Gia Lai province, Vietnam

\begin{tabular}{|c|c|c|c|c|c|c|c|c|c|c|c|c|c|c|c|c|c|}
\hline & \multirow{2}{*}{\multicolumn{2}{|c|}{ Total }} & \multicolumn{14}{|c|}{ By district } & \multirow[t]{3}{*}{$p$-value* } \\
\hline & & & \multicolumn{2}{|c|}{ Chu Puh } & \multicolumn{2}{|c|}{ Chu Se } & \multicolumn{2}{|c|}{ Chu Prong } & \multicolumn{2}{|c|}{ Duc Co } & \multicolumn{2}{|c|}{ la Grai } & \multicolumn{2}{|c|}{ Krong $\mathrm{Pa}$} & \multicolumn{2}{|c|}{ Kong Chro } & \\
\hline & $\mathrm{N}$ & $\%$ & $\mathbf{n}$ & $\%$ & $\mathrm{n}$ & $\%$ & $\mathrm{n}$ & $\%$ & $\mathbf{n}$ & $\%$ & $\mathrm{n}$ & $\%$ & $\mathbf{n}$ & $\%$ & $\mathbf{n}$ & $\%$ & \\
\hline Participants & 3283 & 100 & 600 & 18.3 & 400 & 12.2 & 330 & 10.1 & 530 & 16.1 & 220 & 6.7 & 603 & 18.4 & 600 & 18.3 & $<0.001$ \\
\hline \multicolumn{18}{|l|}{ Age group } \\
\hline $5-9 y$ & 244 & 6.8 & 0 & 0.0 & 0 & 0.0 & 35 & 10.6 & 30 & 5.7 & 20 & 9.1 & 65 & 10.8 & 74 & 12.3 & 0.903 \\
\hline $10-19 y$ & 528 & 16.1 & 134 & 22.3 & 44 & 11.0 & 18 & 5.5 & 52 & 9.8 & 10 & 4.5 & 121 & 20.1 & 149 & 24.8 & 0.057 \\
\hline $20-29 y$ & 1381 & 42.1 & 411 & 68.5 & 354 & 88.5 & 76 & 23.0 & 212 & 40.0 & 55 & 25.0 & 133 & 22.1 & 140 & 23.3 & $<0.001$ \\
\hline $30-39 y$ & 475 & 14.5 & 45 & 7.5 & 1 & 0.3 & 84 & 25.5 & 75 & 14.2 & 38 & 17.3 & 120 & 19.9 & 112 & 18.7 & 0.258 \\
\hline $40-49 y$ & 287 & 8.7 & 10 & 1.7 & 1 & 0.3 & 50 & 15.2 & 60 & 11.3 & 48 & 21.8 & 65 & 10.8 & 53 & 8.8 & 0.412 \\
\hline $50-59 y$ & 211 & 6.4 & 0 & 0.0 & 0 & 0.0 & 27 & 8.2 & 69 & 13.0 & 22 & 10.0 & 59 & 9.8 & 34 & 5.7 & 0.829 \\
\hline $60-65 y$ & 177 & 5.4 & 0 & 0.0 & 0 & 0.0 & 40 & 12.1 & 32 & 6.0 & 27 & 12.3 & 40 & 6.6 & 38 & 6.3 & 0.765 \\
\hline \multicolumn{18}{|l|}{ Sex } \\
\hline Male & 2165 & 65.9 & 599 & 99.8 & 400 & 100.0 & 190 & 57.6 & 346 & 65.3 & 113 & 51.4 & 221 & 36.7 & 296 & 49.3 & $<0.001$ \\
\hline Female & 1118 & 34.1 & 1 & 0.2 & 0 & 0.0 & 140 & 42.4 & 184 & 34.7 & 107 & 48.6 & 382 & 63.3 & 304 & 50.7 & \\
\hline \multicolumn{18}{|l|}{ Ethnicity } \\
\hline Kinh & 912 & 27.8 & 303 & 50.5 & 201 & 50.3 & 162 & 49.1 & 197 & 37.2 & 19 & 8.6 & 1 & 0.2 & 29 & 4.8 & $<0.001$ \\
\hline Ethnic minority & 2371 & 72.2 & 297 & 49.5 & 199 & 49.8 & 168 & 50.9 & 333 & 62.8 & 201 & 91.4 & 602 & 99.8 & 571 & 95.2 & \\
\hline \multicolumn{18}{|l|}{ Occupation } \\
\hline Farmer & 1737 & 52.9 & 0 & 0.0 & 0 & 0.0 & 197 & 59.7 & 393 & 74.2 & 181 & 82.3 & 475 & 78.8 & 491 & 81.8 & $<0.001$ \\
\hline Student & 323 & 9.8 & 0 & 0.0 & 0 & 0.0 & 37 & 11.2 & 33 & 6.2 & 21 & 9.5 & 123 & 20.4 & 109 & 18.2 & 0.227 \\
\hline Gov. worker & 1223 & 37.3 & 600 & 100.0 & 400 & 100.0 & 96 & 29.1 & 104 & 19.6 & 18 & 8.2 & 5 & 0.8 & 0 & 0.0 & $<0.001$ \\
\hline
\end{tabular}

${ }^{*}$ Chi square test 
Table 2 Prevalence of clinical malaria reported by communal health stations for the seven districts in Gia Lai province, Vietnam, 2014 to 2017

\begin{tabular}{|c|c|c|c|c|c|c|c|c|c|c|}
\hline \multirow[t]{2}{*}{ Year } & \multicolumn{3}{|l|}{ Total } & \multicolumn{7}{|c|}{ Prevalence by district (\%) } \\
\hline & Population & $\mathrm{n}$ & $\%$ & Chu Puh & Chu Se & Chu Prong & Duc Co & la Grai & Krong Pa & Kong Chro \\
\hline \multicolumn{11}{|c|}{ a) Population prevalence of clinical malaria } \\
\hline 2014 & 81,346 & 626 & 0.77 & 0.09 & 0.07 & 0.53 & 0.73 & 0.55 & 3.94 & 3.78 \\
\hline 2015 & 85,114 & 300 & 0.35 & 0.04 & 0.02 & 0.28 & 0.36 & 0.10 & 2.08 & 1.72 \\
\hline 2016 & 90,395 & 108 & 0.12 & 0.02 & 0.00 & 0.08 & 0.14 & 0.02 & 0.45 & 0.98 \\
\hline 2017 & 93,184 & 201 & 0.22 & 0.03 & 0.00 & 0.31 & 0.58 & 0.27 & 0.27 & 0.18 \\
\hline \multicolumn{11}{|c|}{ b) Population prevalence of P. falciparum cases } \\
\hline 2014 & 81,346 & 404 & 0.50 & 0.06 & 0.03 & 0.15 & 0.47 & 0.34 & 2.44 & 2.88 \\
\hline 2015 & 85,114 & 174 & 0.20 & 0.03 & 0.02 & 0.23 & 0.08 & 0.06 & 1.33 & 1.24 \\
\hline 2016 & 90,395 & 42 & 0.05 & 0.02 & 0.00 & 0.04 & 0.02 & 0.00 & 0.18 & 0.51 \\
\hline 2017 & 93,184 & 148 & 0.16 & 0.01 & 0.00 & 0.20 & 0.49 & 0.14 & 0.16 & 0.10 \\
\hline \multicolumn{11}{|c|}{ c) Population prevalence of $P$. vivax cases } \\
\hline 2014 & 81,346 & 214 & 0.26 & 0.02 & 0.04 & 0.38 & 0.26 & 0.20 & 1.46 & 0.79 \\
\hline 2015 & 85,114 & 122 & 0.14 & 0.01 & 0.00 & 0.05 & 0.29 & 0.03 & 0.72 & 0.45 \\
\hline 2016 & 90,395 & 64 & 0.07 & 0.00 & 0.00 & 0.04 & 0.13 & 0.02 & 0.27 & 0.44 \\
\hline 2017 & 93,184 & 50 & 0.05 & 0.01 & 0.00 & 0.11 & 0.08 & 0.13 & 0.10 & 0.03 \\
\hline
\end{tabular}

Data provided by Center for Disease Control and Prevention for Gia Lai province, Vietnam [29]

Clinical malaria was defined as symptomatic cases reported by communal health stations with a confirmed microscopy and/or RDT result

\section{Prevalence of asymptomatic malaria}

Two individuals tested positive for malaria by RDT $(0.06 \%, 2 / 3283)$. The prevalence of asymptomatic malaria detected by PCR was $1.74 \%$ (57/3283; P. falciparum $1.07 \%$, P. vivax $0.40 \%$, P. malariae $0.15 \%$, and mixed infections 0.12\%; Table 3). Excluding Chu Puh and Chu Se, which had a different demographic composition and little to no clinical malaria cases, the prevalence of asymptomatic malaria for the other five districts was 2.1\% (49/2283). Asymptomatic individuals were detected in all districts except Chu Se district (Table 3). P. falciparum was the predominant species $(61.4 \%, 35 / 57)$ followed by $P$. vivax $(22.8 \%, 13 / 57)$. The difference in total and individual Plasmodium species malaria distribution was significantly different between the seven districts surveyed. $P$. ovale and $P$. knowlesi malaria were not

Table 3 Prevalence of malaria and Plasmodium species by PCR in the study area, stratified by seven districts in Gia Lai province, Vietnam

\begin{tabular}{|c|c|c|c|c|c|c|c|c|c|}
\hline & \multirow[t]{2}{*}{ Total } & \multicolumn{7}{|l|}{ By district } & \multirow[t]{2}{*}{$p$-value* } \\
\hline & & Chu Puh & Chu Se & Chu Prong & Duc Co & la Grai & Krong Pa & Kong Chro & \\
\hline Samples tested & 3,283 & 600 & 400 & 330 & 530 & 220 & 603 & 600 & \\
\hline \multicolumn{10}{|l|}{$P C R$ result, $n(\%)$} \\
\hline Negative & $3,226(98.3)$ & $592(98.7)$ & $400(100)$ & $327(99.1)$ & $506(95.5)$ & $219(99.6)$ & $588(97.5)$ & 594 (99) & $p<0.001$ \\
\hline Positive & $57(1.7)$ & $\begin{array}{l}8 \\
(1.3)\end{array}$ & $\begin{array}{l}0 \\
(0)\end{array}$ & $\begin{array}{l}3 \\
(0.9)\end{array}$ & $24(4.5)$ & $1(0.5)$ & $15(2.5)$ & $\begin{array}{l}6 \\
(1)\end{array}$ & \\
\hline \multicolumn{10}{|l|}{ Species, $n$ (\%) } \\
\hline$P f$ & $35(61.4)$ & $5(62.5)$ & - & - & $17(70.8)$ & $1(100)$ & $8(53.3)$ & $4(66.7)$ & $p=0.002$ \\
\hline$P_{V}$ & $13(22.8)$ & $\begin{array}{l}2 \\
(25)\end{array}$ & - & $\begin{array}{l}3 \\
(100)\end{array}$ & $7(29.2)$ & - & - & $1(16.7)$ & \\
\hline$P m$ & $5(8.8)$ & - & - & - & - & & $4(26.7)$ & $1(16.7)$ & \\
\hline$P f \& P V$ & $\begin{array}{l}1 \\
(1.8)\end{array}$ & $1(12.5)$ & - & - & - & - & - & - & \\
\hline$P f \& P m$ & $3(5.3)$ & - & - & - & - & - & $3(20)$ & - & \\
\hline
\end{tabular}

P. falciparum - Pf; P. vivax - Pv; P. malariae - Pm; P. falciparum and P. vivax —Pf \& Pv; P. falciparum and P. malariae -Pf \& Pm. ${ }^{*}$ Fisher's exact test 
detected in the participants' blood samples. Out of the 57 PCR positive cases, three presented with clinical malaria within a period of two years after recruitment, including one case of acute vivax malaria seven months after recruitment (personal communication, NVT).

By occupation, PCR positive samples were detected in $2.0 \%(35 / 1737)$ of farmers, $3.1 \%(10 / 323)$ of students and $1.0 \%(12 / 1223)$ of government workers (Additional file 1: Table S2, Fisher's exact $p=0.156)$. No significant differences in PCR malaria prevalence were seen by age group (Additional file 1: Table S3, Fisher's exact $p=0.523$ ), sex (Additional file 1: Table S4, Fisher's exact $p=0.312$ ) or ethnicity (Additional file 1: Table S5, Fisher's exact $p=0.803)$.

\section{Molecular markers of drug resistance for $P$. falciparum malaria}

Molecular markers of drug resistance were analysed in samples with sufficient amount of DNA for PCR amplification and sequencing. No samples had mutations associated with artemisinin resistance in PfKelch-13 (0/13). Among markers associated with piperaquine resistance, none of the samples had the exo-E415G mutation $(0 / 11)$, but $13.3 \%(2 / 15)$ had increased copy numbers of plasmepsin 2/3. Chloroquine resistant haplotype CVIET at positions $72-76$ of $P f c r t$ gene was observed in $27.3 \%$ $(6 / 22)$ samples. Four out of 15 samples (26.7\%) had multiple copies of Pfmdr1. The two isolates with multiple plasmepsin 2/3 copies also had multiple copies of Pfmdr1 gene $(2 / 15,13.3 \%)$.

\section{Serological findings}

Overall, 38.5\% (1257/3262) of participants had antibodies to at least one $P$. falciparum antigen, and $31.1 \%$ (1022/3282) responded to at least one $P$. vivax antigen (Fig. 2 and Additional file 1: Table S6). The highest seroprevalence for individual antigens of each species was $30.6 \%(1001 / 3274)$ for PfAMA1 and $18.7 \%$ (614/3283) for PvAMA1. In contrast, the lowest seroprevalence was found against PfMSP1 (14.4\%, 471/3277) and PvMSP1 (11.1\%, 365/3283), respectively. Seroprevalence varied significantly by district (Additional file 1: Table S6; Pearson's Chi-Square test $p<0.001)$ and were highest in Krong $\mathrm{Pa}$ and Krong Chro districts and lowest in Chu Puh and $\mathrm{Chu}$ Se. Noted, that participants from these latter two districts were predominately male government workers with low clinical malaria rates as well as evidence of low malaria exposure: seroprevalence in Chu Puh and Chu Se was $1 \%$ (6/600) and 4.8\% (19/400) for PfAMA1, and $0.5 \%$ (3/600 and 2/400) for PvAMA1 (Additional file 1: Table S6). Due to the age and gender homogeneity these two districts were excluded from subsequent analysis.
Factors associated with $P$. falciparum and $P$. vivax exposure (defined as being seropositive to any antigen for each species) were determined using logistic regression in five of the study districts (Table 4). In these areas, 52\% of the participants had evidence of previous exposure to P. falciparum (1178/2279) and 42\% (962/2282) to P. vivax. There was no association between PCR positivity ( $P$. falciparum: OR 0.88 [95\% CI 0.50-1.55], $p=0.660$; P. vivax: OR 0.72 [95\% CI 0.40-1.31], $p=0.287$ ) and antibody responses. Female sex, being from an ethnic minority group, farming or residing in Krong Pa and Kong Chro districts indicated an increased risk of both $P$. falciparum and $P$. vivax exposure $(p<0.001)$. Overall, there was an increased risk of seropositivity for older age groups as compared to young children, especially for P. falciparum.

Seroprevalence data were used to estimate malaria transmission intensity by fitting age-seroprevalence curves for $P$. falciparum and $P$. vivax exposure at each district (Figs. 3 and 4), as well as for response to individual antigens (Additional file 1: Figure S2 for P. falciparum antigens and Additional file 1: Figure S3 for $P$. vivax antigens). Seroconversion rates were highest in Krong Pa and Kong Chro ( $\lambda$ range for $P$. falciparum $=0.063-0.162$; $\lambda$ range for $P$. vivax $=0.066-0.223)$, the districts with the highest prevalence of clinical malaria in 2014-2015 (Figs. 3 and 4, Additional file 1: Table S1 and Additional file 1: Table S7). In contrast, seroconversion was lower in Chu Prong, Duc Co and Ia Grai districts ( $\lambda$ range for $P$. falciparum $=0.009-0.031 ; \lambda$ range for $P$. vivax $=0.004-$ 0.008; Additional file 1: Table S7). In these three districts, responses elicited against PfAMA1 and PvAMA1 were the main contributors to overall exposure data, as the number of individuals seropositive for MSP1 and/or CSP antigens was low (Additional file 1: Figure S2 and Additional file 1: Figure S3).

\section{Discussion}

Sub-clinical malaria represents a disease reservoir that should be considered when determining the malaria risk for naïve people, and for developing malaria control and elimination strategies in areas transitioning from preelimination to elimination. This is the first study to provide a snapshot of the burden of asymptomatic malaria in Gia Lai province, Vietnam.

The overall prevalence of asymptomatic malaria in the 3283 participants was $1.74 \%$ in December $2016-$ January 2017, with $96.4 \%$ (55/57) below the RDT detection threshold. This prevalence was markedly higher than the mean clinical malaria prevalence rate of $0.12 \%$ $(108 / 90,395)$ reported in 2016 among residents from the 14 communes where the participants were recruited. Although the survey included a subset of the province population, other studies in similar epidemiological 


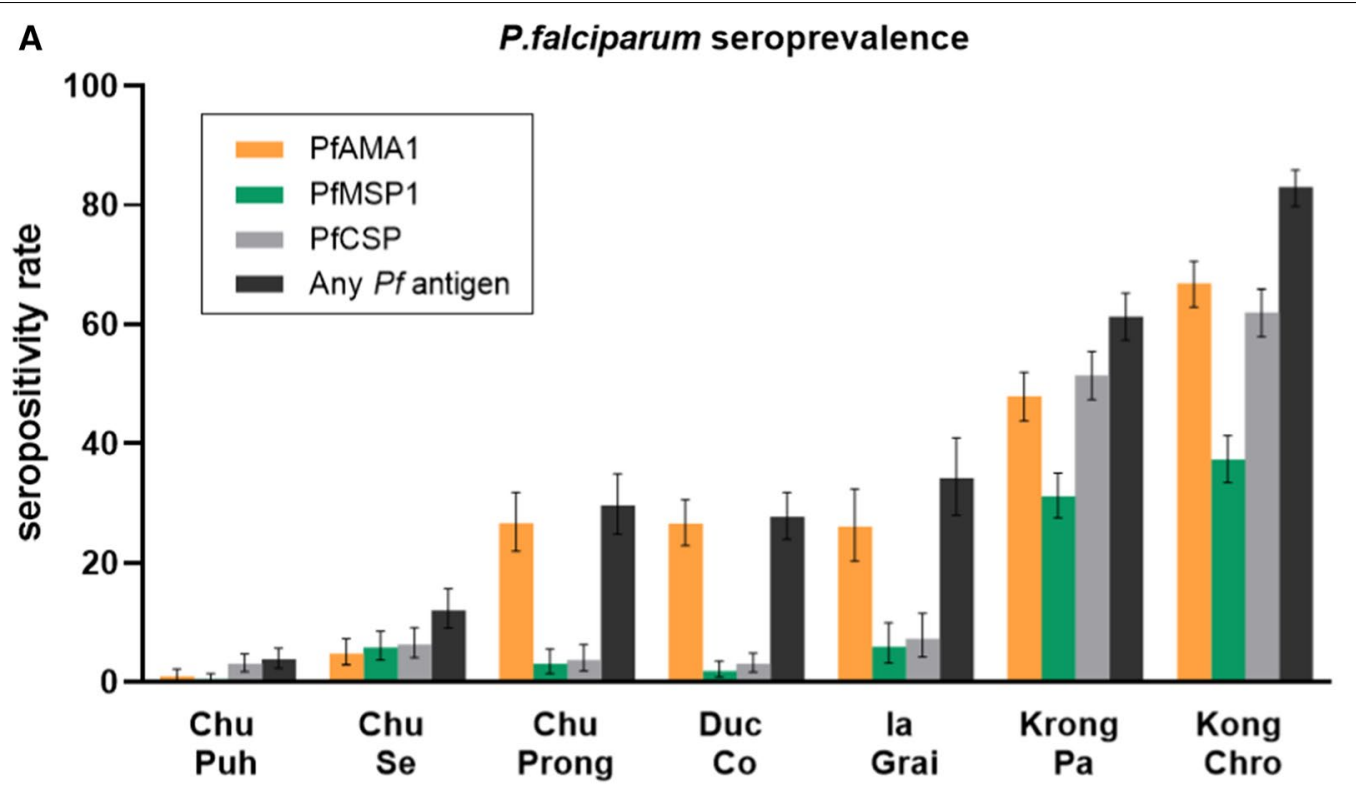

B

\section{P.vivax seroprevalence}

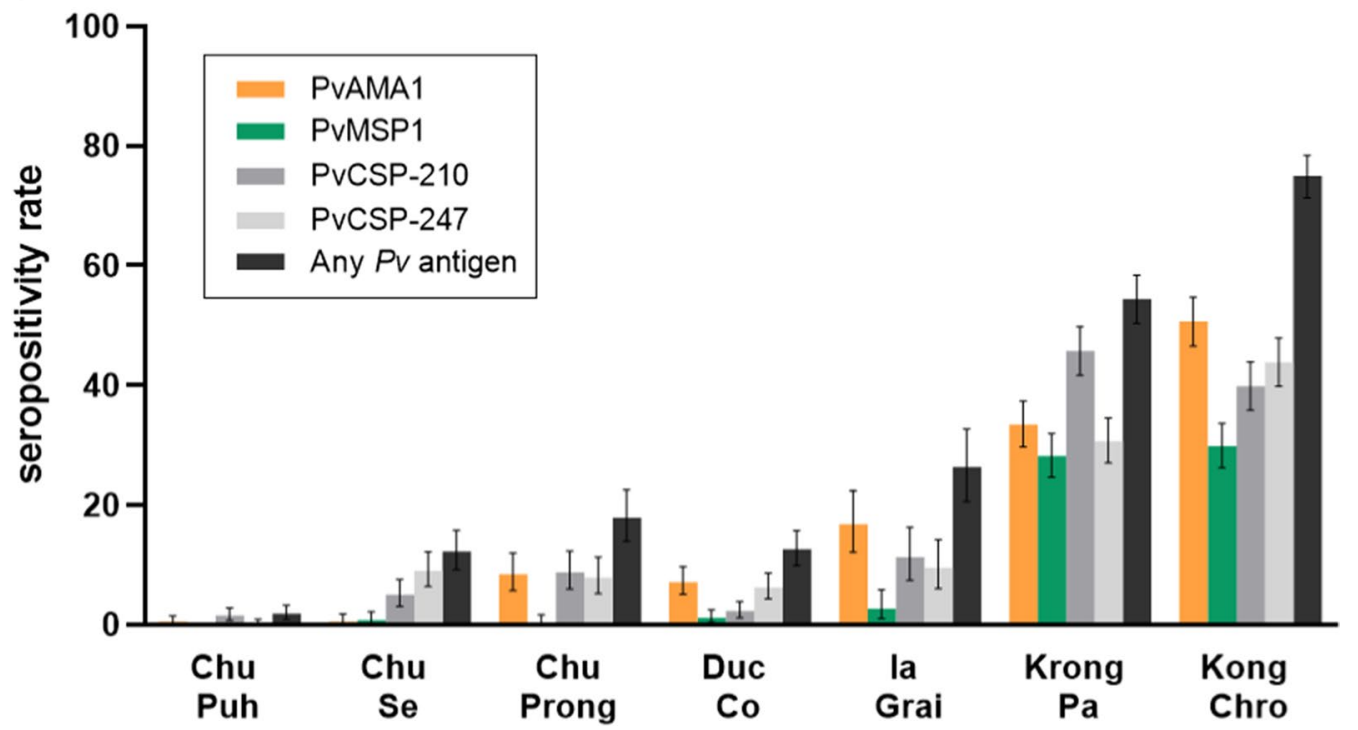

Fig. 2 Seroprevalence of participants in seven districts in Gia Lai province against P. falciparum and P. vivax antigens. Error bars indicate $95 \% \mathrm{Cl}$

settings have indicated that asymptomatic Plasmodium carriers are substantially more prevalent than clinical cases $[8,9]$. Moreover, the district selection covered geographically distinct areas, as well as people from all age groups, minority ethnicities, and different occupations. In the case of Chu Puh and Chu Se districts the recruitment included mainly adults from military forces, which could suggest a bias in the asymptomatic malaria prevalence estimates. However, both districts are semi-urban areas where a decrease in overall malaria cases has been observed in the past years and thus a lower prevalence of asymptomatic infections could be expected.

Importantly, the PCR assay in the present study using a finger prick capillary blood of approximately $10 \mu \mathrm{L}$ and based on the PCR performance acceptance threshold was designed to detect sub-microscopic malaria infections with a sensitivity of at least 1.6 to eightfold greater than that of microscopy (the LOD in thick blood films for 
Table 4 Factors associated with P. falciparum and P. vivax seropositivity to any antigen in the study area, Gia Lai province (excluding Chu Puh and Chu Se districts)

\begin{tabular}{|c|c|c|c|c|c|c|c|c|}
\hline \multirow[t]{3}{*}{ Variable } & \multicolumn{4}{|c|}{ Any $P$. falciparum antigen } & \multicolumn{4}{|c|}{ Any $P$. vivax antigen } \\
\hline & \multicolumn{2}{|c|}{ Seropositivity } & \multirow[t]{2}{*}{ OR $(95 \% \mathrm{Cl})$} & \multirow[t]{2}{*}{$p$-value* } & \multicolumn{2}{|c|}{ Seropositivity } & \multirow[t]{2}{*}{ OR $(95 \% \mathrm{Cl})$} & \multirow[t]{2}{*}{$p$-value } \\
\hline & $\mathrm{n} / \mathrm{N}$ & Rate (\%) & & & $\mathrm{n} / \mathrm{N}$ & Rate (\%) & & \\
\hline \multicolumn{9}{|l|}{$P C R$} \\
\hline Negative & $1163 / 2230$ & 52.1 & & & $945 / 2233$ & 42.3 & & \\
\hline Positive & $24 / 49$ & 48.9 & $0.88(0.5-1.55)$ & 0.660 & $17 / 49$ & 34.7 & $0.72(0.40-1.31)$ & 0.287 \\
\hline \multicolumn{9}{|l|}{ Age group } \\
\hline $5-9 y$ & $90 / 223$ & 40.4 & & & $77 / 224$ & 34.4 & & \\
\hline $10-19 y$ & $11 / 20$ & 55.0 & $1.81(0.72-4.54)$ & 0.208 & $12 / 20$ & 60.0 & $2.86(1.12-7.3)$ & 0.028 \\
\hline $20-29 y$ & $31 / 116$ & 26.7 & $0.54(0.33-0.88)$ & 0.014 & $26 / 116$ & 22.4 & $0.55(0.33-0.92)$ & 0.024 \\
\hline $30-39 y$ & $46 / 75$ & 61.3 & $2.34(1.37-4.01)$ & 0.002 & $38 / 74$ & 51.3 & $2.01(1.18-3.43)$ & 0.010 \\
\hline $40-49 y$ & $38 / 62$ & 61.3 & $2.34(1.31-4.17)$ & 0.004 & $29 / 62$ & 46.8 & $1.68(0.94-2.97)$ & 0.075 \\
\hline $50-59 y$ & $17 / 34$ & 50.0 & $1.48(0.72-3.04)$ & 0.290 & $15 / 34$ & 44.1 & $1.51(0.73-3.13)$ & 0.271 \\
\hline $60-69 y$ & 109/176 & 61.9 & $2.4(1.6-3.61)$ & $<0.001$ & $88 / 177$ & 49.7 & $1.89(1.26-2.83)$ & 0.002 \\
\hline \multicolumn{9}{|l|}{ Sex } \\
\hline Male & $529 / 1164$ & 45.4 & & & $436 / 1166$ & 37.4 & & \\
\hline Female & $658 / 1115$ & 59.0 & $1.73(1.46-2.04)$ & $<0.001$ & $526 / 1116$ & 47.1 & $1.49(1.26-1.73)$ & $<0.001$ \\
\hline \multicolumn{9}{|l|}{ Ethinicity } \\
\hline Kinh & $91 / 408$ & 22.3 & & & $67 / 408$ & 16.4 & & \\
\hline Minority & 1096/1871 & 58.6 & $4.93(3.83-6.33)$ & $<0.001$ & $895 / 1874$ & 47.8 & $4.65(3.53-6.14)$ & $<0.001$ \\
\hline \multicolumn{9}{|l|}{ District } \\
\hline Chu Prong & $97 / 327$ & 29.7 & & & $59 / 329$ & 17.9 & & \\
\hline Duc Co & $147 / 530$ & 27.7 & $0.91(0.67-1.23)$ & 0.544 & $67 / 530$ & 12.6 & $0.66(0.45-0.97)$ & 0.034 \\
\hline la Grai & $75 / 219$ & 34.2 & $1.23(0.86-1.78)$ & 0.259 & $58 / 220$ & 26.4 & $1.64(1.09-2.47)$ & 0.019 \\
\hline Krong Pa & $370 / 603$ & 61.4 & $3.76(2.82-5.02)$ & $<0.001$ & $328 / 603$ & 54.4 & $5.46(3.95-7.54)$ & $<0.001$ \\
\hline Kong Chro & $498 / 600$ & 83.0 & $11.58(8.42-15.92)$ & $<0.001$ & $450 / 600$ & 75.0 & $13.73(9.8-19.22)$ & $<0.001$ \\
\hline \multicolumn{9}{|l|}{ Occupation } \\
\hline Farmer & $1015 / 1734$ & 58.5 & & & 798/1736 & 46.0 & & \\
\hline School age & $142 / 322$ & 44.1 & $0.56(0.44-0.71)$ & $<0.001$ & $138 / 323$ & 42.7 & $0.88(0.69-1.11)$ & 0.283 \\
\hline Worker/Soldier & $30 / 223$ & 13.5 & $0.11(0.07-0.16)$ & $<0.001$ & $26 / 223$ & 11.7 & $0.16(0.1-0.24)$ & $<0.001$ \\
\hline
\end{tabular}

* Univariate logistic regression

expert microscopists is estimated at 4 to 20 parasites $/ \mu \mathrm{L}$ [30]). Moreover, we cannot exclude that the sub-microscopic reservoir was larger if using alternate molecular methods that offered greater sensitivity, such as ultrasensitive quantitative PCR assays that use high blood volume $(2-3 \mathrm{~mL}$ of venous blood; LOD as low as 22 parasites $/ \mathrm{mL}$ ) $[9,31]$, or assays targeting multi-copy gene families (LOD 30-150 parasites/mL) [32]. In this study, finger-prick capillary blood collection followed by standard PCR was selected to minimize the burden on study participants (reluctance to provide venous blood) and reduce logistic requirements and costs, both of which would have impacted on the conduct of present study as well as potential feasibilities of future surveys.

The results suggest no presence of $P$. ovale and $P$. knowlesi infections in the study population. In the present study, the PCR assay sensitivity was only tested for P. falciparum. Although rare, P. ovale infection detected by PCR has been reported in several southern Vietnam provinces (Song Be, Lam Dong, Dak Lak, and Khanh Hoa) [33] and in south-central Ninh Thuan province [34], but the species has not been reported in Gia Lai yet. Similarly, P. knowlesi has been reported in south-central Ninh Thuan province [35] and in central Quang Tri province [36], but not in Gia Lai. The detection of cases of asymptomatic $P$. malariae (three mixed infections of $P$. falciparum and $P$. malariae, and five mono-infections of $P$. malariae) supports other reports of cryptic or asymptomatic $P$. malariae infections in Southeast Asia [37]. The clinical and public health importance of these asymptomatic infections is unknown, but the prevalence may be sufficient to maintain transmission in the study area and warrants further investigation. 

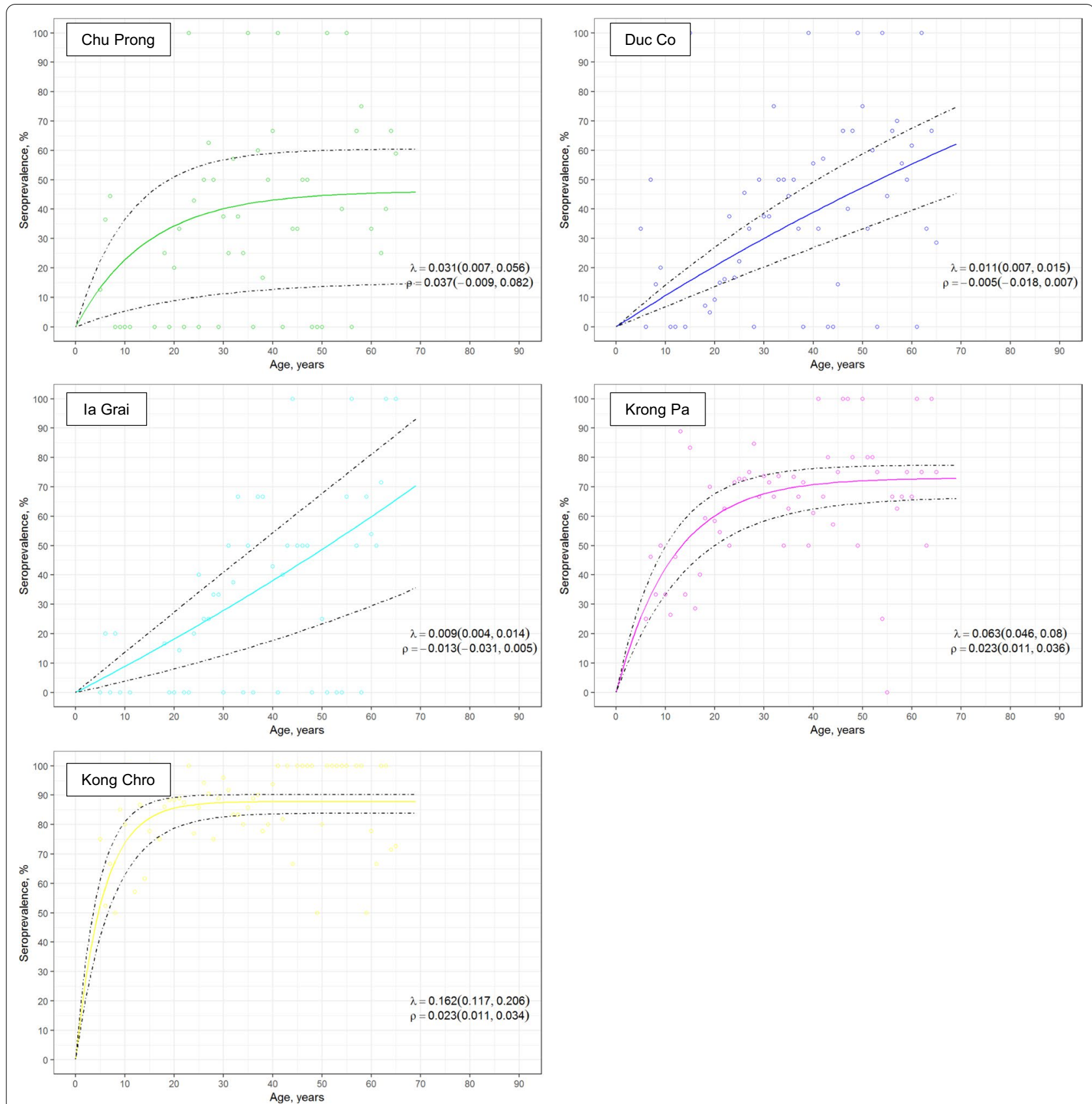

Fig. 3 Age-seroprevalence curves for P. falciparum exposure across five districts in Gia Lai province, Vietnam. Exposure is defined as seropositivity to at least one $P$. falciparum antigen. Reversible catalytic conversion models allowing one seroconversion rate $(\lambda)$ were fit to the data (dashed line indicates $95 \% \mathrm{Cl}$ )

Among asymptomatic infections caused by $P$. falciparum no Pfkelch-13 mutations that confer artemisinin resistance were detected $(0 / 13)$. This finding contrasts with other recent studies that reported a prevalence of Pfkelch-13 validated mutations of $74 \%(20 / 27)$ in Ia Grai district in 2015, and up to 89\% (39/45) in Krong $\mathrm{Pa}$ district in 2017, in both cases C580Y mutations were predominant $[19,20]$. Unlike the present study, both studies by Thanh et al. and Rovira-Vallbona et al. were clinical trials conducted with symptomatic individuals presenting with high parasite densities $(>500$ parasites $/ \mu \mathrm{L})[19,20]$. The absence of PfKelch-13 mutations in samples collected from asymptomatic individuals in the present study could be due to the small 

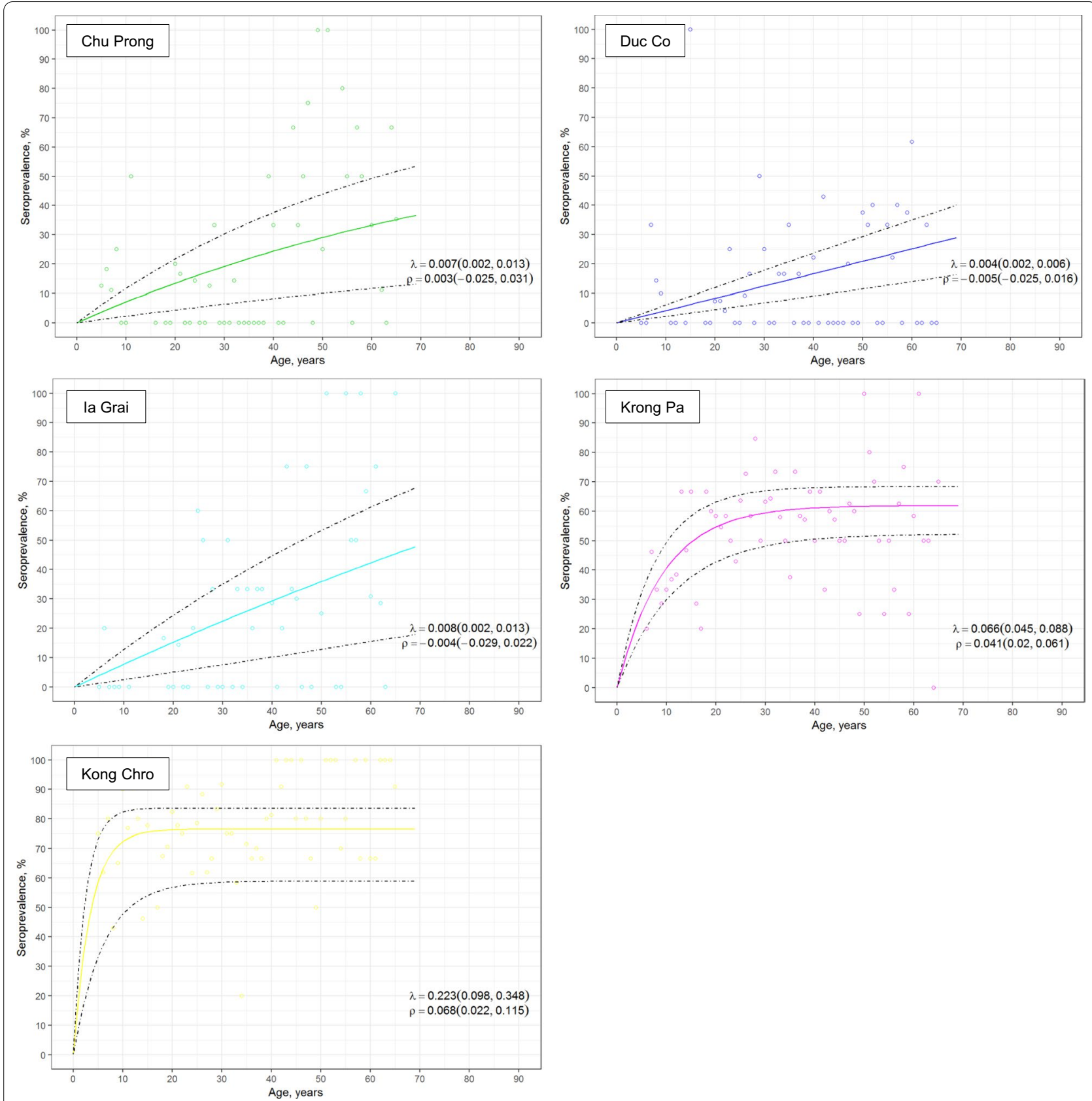

Fig. 4 Age-seroprevalence curves for P. vivax exposure across five districts in Gia Lai province, Vietnam. Exposure is defined as seropositivity to at least one P. vivax antigen. Reversible catalytic conversion models allowing one seroconversion rate $(\lambda)$ were fit to the data (dashed line indicates $95 \%$ $\mathrm{Cl})$

sample size of the subset of $P$. falciparum positive samples $(13 / 35)$ for which the sequence data were obtained and/or heterogeneity in the local parasite populations. Another possible explanation is that in the absence of drug pressure resistant parasites appear to be less fit in comparison with the wild type parasites [38], and thus could be outgrown by the wild type parasites in low parasitemia asymptomatic infections. On the other hand, multiple copies of the plasmepsin $2 / 3$ genes and pfmdr1 gene were found in $13.3 \%,(2 / 15)$ and $26.7 \%$ $(4 / 15)$ of infections, respectively, including two double mutants. Amplification of plasmepsin 2/3 confer resistance to piperaquine, the partner drug in the firstline artemisinin-combination therapy DHA-PPQ used 
in Vietnam up until 2020. The rate of plasmepsin $2 / 3$ amplification coincides with that reported in a clinical trial conducted Krong Pa district during the same period in Vietnamese subjects $(10.4 \%, 5 / 48)$ [20]. Increases in the $p f m d r 1$ copy number are associated with resistance to mefloquine, which has not been used in the study area since the 1990s (personal communication with Dr. Ngo Duc Thang). This suggests the possibility of importation of resistant malaria parasite from neighboring Cambodia, where artesunate-mefloquine has been used to treat $P$. falciparum malaria in early 2000s [39]. Further investigation is needed to better understand the movement of individuals carrying malaria parasites across the international border that Gia Lai province shares with Cambodia.

This study also aimed to explore serology as an alternative malaria transmission metric for surveillance purposes. The antigens AMA-1, MSP1 and CSP, were selected as they are present in both species, represent both pre-erythrocytic and erythrocytic stage parasites and have been extensively used as markers of exposure in the past. The analysis of antibody responses of study participants found that 38.5\% (1257/3262) and 31.1\% $(1022 / 3282)$ of individuals responded to at least one $P$. falciparum or $P$. vivax antigen, respectively. Although no other serological surveys had been conducted previously in Gia Lai, these rates compare to those found in similar epidemiological settings, such as a population survey in Quang Nam, Vietnam, in 2015 (42.3\%, 145/343 for $P$. falciparum and $22.4 \%, 77 / 343$ for $P$. vivax), or a study among malaria-negative adults in Oddar Meanchey, Cambodia, in 2011 (50\%, 57/113 for P. falciparum-MSP1 and $61 \%, 69 / 113$ for $P$. vivax-MSP1) [18, 40]. The relatively high seroprevalence rates as compared to parasitological estimates could be explained by (1) the fact that antibody responses reflect cumulative exposure events in the past, including asymptomatic infections, (2) the relatively long half-life (i.e. $>6$ months) of responses against Pf/PvAMA1 or Pf/PvMSP1 antigens generated after exposure events [41, 42] and, to a minor extent, (3) missed sub-microscopic infections in the crosssectional survey that could increase population malaria prevalence rate. Antibody decay rates (or half-lives) for the antigens used in the present study had been previously determined in a cohort from Ratanakiri province, Cambodia (bordering Gia Lai province) using multiple antibody measurements over 2 years' time; the authors reported half-lives to be between $\approx 10$ and 28 months in individuals that remained PCR negative during the follow-up (i.e. 297 days for PfMSP1, 569 for PfCSP, 367 for PvAMA1, 312 for PvMSP1, 885 for PvCSP210 and 725 for PvCSP247) [42]. The long half-live against CSP, particularly for the two $P$. vivax antigens, may partly explain the high seroprevalence found for CSP antigens in some of the districts included in the present study.

Age-seroprevalence curves allowed to identify two types of districts. For Chu Prong, Duc Co, and Ia Grai districts a linear association between age and seroprevalence rate was seen, suggestive of a sustained decrease in malaria prevalence in the past years. In contrast, Krong $\mathrm{Pa}$ and Kong Chro districts had the highest seroconversion rates for all antigens and a rapid acquisition of antibodies at early ages for both $P$. falciparum and $P$. vivax antigens, what can be interpreted as ongoing transmission of both species. Although the higher number of individuals $<20$ years of age in Krong Pa and Kong Chro might have contributed to these differences, retrospective data showed these two districts had the highest clinical malaria rate among study districts in the two years preceding the survey, in line with serological results. The decrease in malaria cases reported between 2014 and 2016 in all districts by an average of 6.4-fold was not captured in the age-seroprevalence curves conducted at the end of 2016, probably due to the high immunogenicity and long duration of responses to selected antigens.

Risk factors for seropositivity were assessed after excluding the two districts with only military personnel and negligible seropositivity rates (i.e. Chu Puh and Chu $\mathrm{Se})$. Even so, district of residence significantly increased risk of seropositivity for both Plasmodium species, in agreement with the higher number of malaria cases that detected in Krong Pa and Kong Chro communal health stations in recent years (Tables 2 and 4). Plasmodium falciparum antibody prevalence was also highest among older age groups, what may be explained by different risk behaviour compared to younger student-age groups (i.e. working in farming and sleeping outdoors) together with their history of exposure to infection during their lifetime. Similarly, the higher seroprevalence in females than males may be due to a higher proportion of females being farmers by occupation (83.8\%; 936/1117) compared with males $(68.7 \%$; 801/1166) in these communities. Farming activities often include going far into forests where risk of malaria is higher. On the other hand, no association was seen between PCR positivity in the cross-sectional survey and $P$. falciparum or $P$. vivax seroprevalence. Although studies in low endemic areas have shown that even low-density asymptomatic infections can induce strong long-lived IgG responses against $P$. vivax [43], the number of PCR positive individuals was low. Another study using qPCR for parasite diagnosis in Quang Nam province could not identify active parasite carriers despite serological evidence of ongoing transmission in consecutive cross-sectionals [18]. As compared to parasite prevalence surveys that can only identify active infections, serological surveys in pre-elimination settings 
can be cost-effective tools to confirm the cease in transmission in a given area, and/or to measure changes in transmission intensity over time.

\section{Conclusions}

Sub-clinical malaria represents a disease reservoir that should be considered when planning malaria elimination strategies and for determining the malaria risk for naïve personnel, such as travelers and military personnel visiting malaria endemic areas. In this study, the asymptomatic prevalence of malaria infections was $1.74 \%$ in seven districts of Gia Lai province, an area of low clinical malaria endemicity. The high seropositivity rates for Plasmodium antigens suggest that a significant proportion of participants had previous malaria exposure, and age-dependent trends allowed to differentiate districts with sustained low transmission from others with higher ongoing transmission levels of both $P$. falciparum and $P$. vivax. The results of this study can inform NMCP on the burden of asymptomatic malaria in Gia Lai and provide tools for geographical stratification of transmission, in order to design efficient public health and elimination strategies.

\begin{abstract}
Abbreviations
Cl: Confidence interval; DHA-PPQ: Dihydroartemisinin-piperaquine; ELISA: Enzyme-linked immunosorbent assay; GMS: Greater Mekong Subregion; ITN: Insecticide-treated net; LOD: Limit of detection; NIMPE: National Institute of Malariology, Parasitology and Entomology; NMCP: National Malaria Control Programme; OD: Optical density; OR: Odds ratio; PBS: Phosphate buffered saline; PCR: Polymerase chain reaction; PQR: Prevalence odds ratios; qPCR: Quantitative real-time PCR; RDT: Rapid diagnostic test; WHO: World Health Organization.
\end{abstract}

\section{Supplementary Information}

The online version contains supplementary material available at https://doi. org/10.1186/s12936-022-04060-6.

Additional file 1: Figure S1. Reproducibility of the ELISA assays for the seven Plasmodium antigens for $15 \%$ of samples $(n=575)$. Table $\mathbf{S 1}$. Malaria prevalence for blood film and/or rapid diagnostic testing positive people in the seven districts of Gia Lai province, Vietnam. Table S2. PCR results by Plasmodium species and self-reported occupation of study subjects from Gia Lai province, Vietnam. Table S3. PCR result and Plasmodium species by age-group of study subjects from Gia Lai province, Vietnam. Table S4. PCR result and Plasmodium species by sex of study subjects from Gia Lai province, Vietnam. Table S5. PCR result and Plasmodium species by ethnicity of study subjects from Gia Lai province, Vietnam. Table S6. Seroprevalence against $P$. falciparum and $P$. vivax by districts of study subjects from Gia Lai province, Vietnam. Table S7. Seroconversion rates by districts of study subjects from Gia Lai province, Vietnam. Figure S2. Age-seroprevalence curves for individual P. falciparum antigens by districts of study subjects from Gia Lai province, Vietnam. Reversible catalytic conversion models allowing one seroconversion rate $(\lambda)$ were fit to the data (dashed line shows $95 \% \mathrm{Cl}$ ). Figure $\mathbf{S 3}$. Age-seroprevalence curves for individual P. vivax antigens by districts of study subjects from Gia Lai province, Vietnam. Reversible catalytic conversion models allowing one seroconversion rate $(\lambda)$ were fit to the data (dashed line shows $95 \% \mathrm{Cl}$ ).

\section{Acknowledgements}

We would like to thank the participants who volunteered their time to be in the study. Additionally, the authors are grateful to Dr. Nguyen Xuan Tien, Dr. Pham Xuan Vinh, Dr. Phan Tan Dan, Ms. Nguyen Thi Minh Phuong, and Dr. Nguyen Thi Thanh Van from the Military Institute of Preventive Medicine (MIPM) for their coordination of field work and ensuring all participants were fully informed of the study purpose; Mr. Ro Mah Huan from Center for Disease Control and Prevention, Gia Lai province; Dr. Nguyen Chinh Phong, Dr. Bui Duc Hai and Mr. Nguyen Thanh Hai from Military Medical Department (Ministry of Defense, Vietnam); Dr. Vincent Sluydts from University of Antwerp (Belgium); and Dr. Gerard C. Kelly from Vysnova Partners, Inc.

\section{Disclaimer}

The views expressed in this article are those of the authors and do not necessarily reflect the official policy or position of the Department of the Navy, the Department of Defense or the U.S. government and also those of the Australian Defence Force, Joint Health Command or any extant Australian Defence Force policy. For KAE and NJM: I am a military Service member. This work was prepared as part of my official duties. Title 17, U.S.C., \$105 provides that copyright protection under this title is not available for any work of the U.S. Government. Title 17, U.S.C., \$101 defines a U.S. Government work as a work prepared by a military Service member or employee of the U.S. Government as part of that person's official duties.

\section{Authors' contributions}

NDM and NNS made equal contribution to the study. NJM, NNS, NDM, and MDE designed the study and developed the protocol. NXK obtained approval of military health facilities to support the cross-sectional survey. NNS and NJM obtained scientific and ethical approval for the study. NNS, NVT and TKL carried out the field work. MC provided direction, training, and quality assurance of the PCR assays. NHB performed the serological assays with scientific contribution from KAE. ERV and NHB analysed the serological data and ERV critically reviewed the serological findings. NJM, ERV, MC and MDE wrote the manuscript. All authors read and approved the final manuscript.

\section{Funding}

This work was funded by the US Defense Health Program-Research, Development, Testing and Evaluation (work unit number D1430).

\section{Availability of data and materials}

The datasets generated during and/or analysed during the current study are available from the corresponding author on reasonable request.

\section{Declarations}

\section{Ethics approval and consent to participate}

Ethics review was conducted by the Vietnam National Institute of Malaria, Parasitology and Entomology Institutional Review Board (2016-VM-P5) and extramural review was conducted in accordance with U.S. Department of the Navy Human Research Protection Program guidelines (HRPO.NMRCA.2017.0003) in compliance with all applicable federal regulations governing the protection of human subjects. Written informed consent was obtained from participants and parents or guardians of minor participants prior to enrolment. Children aged 10 to 17 years of age provided assent to participate in the study.

\section{Consent for publication}

Not applicable.

\section{Competing interests}

The authors declare that they have no competing interest.

\section{Author details}

${ }^{1}$ Hanoi Medical University, Hanoi, Vietnam. ${ }^{2}$ Vietnam People's Army Military Medical Department, Hanoi, Vietnam. ${ }^{3}$ Vietnam People's Army Military Institute of Preventive Medicine, Hanoi, Vietnam. ${ }^{4}$ Australian Defence Force Malaria and Infectious Disease Institute, Brisbane, Australia. ${ }^{5}$ National Institute of Malariology Parasitology and Entomology, Hanoi, Vietnam. ${ }^{6}$ Vysnova Partners, Bethesda, MD, USA. ${ }^{7}$ U.S. Naval Medical Research Unit TWO, Singapore, Singapore. 
Received: 13 July 2021 Accepted: 23 January 2022

Published online: 08 February 2022

\section{References}

1. WHO. Malaria in the Western Pacific Region. Manila, Western Pacific Regional World Health Organization, 2018.

2. NIMPE. National strategy for malaria control and elimination in the period 2011-2020 and orientation to 2030. Vietnam National Institute of Malariology, Parasitology, and Entomology, 2011

3. Smith DL, Cohen JM, Chiyaka C, Johnston G, Gething PW, Gosling R, et al. A sticky situation: the unexpected stability of malaria elimination. Philos Trans R Soc Lond B Biol Sci. 2013;368:20120145.

4. WHO. Emergency response to artemisinin resistance in the Greater Mekong subregion. Regional framework for action 2013-2015. Geneva, World Health Organization, 2013.

5. Wangdi K, Canavati SE, Ngo TD, Tran LK, Nguyen TM, Tran DT, et al. Analysis of clinical malaria disease patterns and trends in Vietnam 2009-2015. Malar J. 2018:17:332

6. Marchand RP, Culleton R, Maeno Y, Quang NT, Nakazawa S. Co-infections of Plasmodium knowlesi, P. falciparum, and P. vivax among humans and Anopheles dirus mosquitoes, Southern Vietnam. Emerg Infect Dis. 2011:17:1232-9.

7. Eede PVd, Van HN, Van Overmeir C, Vythilingam I, Duc TN, Hung LX, et al. Human Plasmodium knowlesi infections in young children in central Vietnam. Malar J. 2009;8:249.

8. Imwong M, Nguyen TN, Tripura R, Peto TJ, Lee SJ, Lwin KM, et al. The epidemiology of subclinical malaria infections in South-East Asia: findings from cross-sectional surveys in Thailand-Myanmar border areas, Cambodia, and Vietnam. Malar J. 2015:14:381.

9. Phommasone K, Adhikari B, Henriques G, Pongvongsa T, Phongmany P, von Seidlein $L$, et al. Asymptomatic Plasmodium infections in 18 villages of southern Savannakhet Province, Lao PDR (Laos). Malar J. 2016;15:296.

10. Endeshaw T, Gebre T, Ngondi J, Graves PM, Shargie EB, Ejigsemahu Y, et al. Evaluation of light microscopy and rapid diagnostic test for the detection of malaria under operational field conditions: a household survey in Ethiopia. Malar J. 2008;7:118.

11. Corran P, Coleman P, Riley E, Drakeley C. Serology: a robust indicator of malaria transmission intensity? Trends Parasitol. 2007;23:575-82.

12. Bretscher MT, Supargiyono S, Wijayanti MA, Nugraheni D, Widyastuti AN, Lobo NF, et al. Measurement of Plasmodium falciparum transmission intensity using serological cohort data from Indonesian schoolchildren. Malar J. 2013;12:21.

13. O'Meara WP, Collins WE, McKenzie FE. Parasite prevalence: a static measure of dynamic infections. Am J Trop Med Hyg. 2007:77:246-9.

14. Greenhouse B, Daily J, Guinovart C, Goncalves B, Beeson J, Bell D, et al. Priority use cases for antibody-detecting assays of recent malaria exposure as tools to achieve and sustain malaria elimination. Gates Open Res. 2019:3:131.

15. Rogier E, Wiegand R, Moss D, Priest J, Angov E, Dutta S, et al. Multiple comparisons analysis of serological data from an area of low Plasmodium falciparum transmission. Malar J. 2015;14:436.

16. Drakeley CJ, Corran PH, Coleman PG, Tongren JE, McDonald SL, Carneiro I, et al. Estimating medium- and long-term trends in malaria transmission by using serological markers of malaria exposure. Proc Natl Acad Sci USA. 2005:102:5108-13.

17. Arnold BF, Priest JW, Hamlin KL, Moss DM, Colford JM Jr, Lammie PJ. Serological measures of malaria transmission in Haiti: comparison of longitudinal and cross-sectional methods. PLoS ONE. 2014;9:e93684.

18. Kattenberg JH, Erhart A, Truong MH, Rovira-Vallbona E, Vu KAD, Nguyen THN, et al. Characterization of Plasmodium falciparum and Plasmodium vivax recent exposure in an area of significantly decreased transmission intensity in Central Vietnam. Malar J. 2018;17:180

19. Thanh NV, Thuy-Nhien N, Tuyen KN, Tong TN, Nha-Ca TN, Dong TL, et al. Rapid decline in the susceptibility of Plasmodium falciparum to dihydroartemisinin-piperaquine in the south of Vietnam. Malar J. 2017;16:27.

20. Rovira-Vallbona E, Hong NV, Kattenberg JH, Huan RM, Hien NTT, Ngoc $\mathrm{NTH}$, et al. Efficacy of dihydroartemisinin/piperaquine and artesunate monotherapy for the treatment of uncomplicated Plasmodium falciparum malaria in Central Vietnam. J Antimicrob Chemother. 2020;75:2272-81.
21. Padley D, Moody AH, Chiodini PL, Saldanha J. Use of a rapid, single-round, multiplex PCR to detect malaria species and identify species present. Ann Trop Med Parasitol. 2003;97:131-7.

22. Imwong M, Tanomsing N, Pukrittayakamee S, Day NP, White NJ, Snounou G. Spurious amplification of a Plasmodium vivax small-subunit RNA gene by use of primers currently used to detect P. knowlesi. J Clin Microbiol. 2009:47:4173-5.

23. WHO. Status report on artemisinin resistance and ACT efficacy. Geneva, World Health Organization, 2018. https://www.who.int/malaria/publi cations/atoz/artemisinin-resistance-august2018/en/

24. Ariey F, Witkowski B, Amaratunga C, Beghain J, Beghain J, Langlois AC, et al. A molecular marker of artemisinin-resistant Plasmodium falciparum malaria. Nature. 2014;505:50-5.

25. Amato R, Lim P, Miotto O, Amaratunga C, Dek D, Pearson RD, et al. Genetic markers associated with dihydroartemisinin-piperaquine failure in Plasmodium falciparum malaria in Cambodia: a genotype-phenotype association study. Lancet Infect Dis. 2017;17:164-73.

26. Witkowski B, Duru V, Khim N, Ross LS, Saintpierre B, Beghain J, et al. A surrogate marker of piperaquine-resistant Plasmodium falciparum malaria: a phenotype-genotype association study. Lancet Infect Dis. 2016;17:174-83.

27. Chen N, Kyle DE, Pasay C, Fowler EV, Baker J, Peters JM, Cheng Q. pfort allelic types with two novel amino acid mutations in chloroquineresistant Plasmodium falciparum isolates from the Philippines. Antimicrob Agents Chemother. 2003;47:3500-5.

28. Chavchich M, Gerena L, Peters J, Chen N, Cheng Q, Kyle DE. Role of pfmdr 1 amplification and expression in induction of resistance to artemisinin derivatives in Plasmodium falciparum. Antimicrob Agents Chemother 2010:54:2455-64.

29. Center for Disease Control and Prevention (CDC) for Dak Lak Province Vietnam. 2020. Communal health station annual malaria cases based on positive blood film microscropy and/or rapid diagnostic testing by districts in Dak Lak Province for 2014 to 2017.

30. Wongsrichanalai C, Barcus MJ, Muth S, Sutamihardja A, Wernsdorfer WH. A review of malaria diagnostic tools: microscopy and rapid diagnostic test (RDT). Am J Trop Med Hyg. 2007;77(6 Suppl):119-27.

31. Imwong M, Hanchana S, Malleret B, Rénia L, Day NP, Dondorp A, et al. High-throughput ultrasensitive molecular techniques for quantifying low-density malaria parasitemias. J Clin Microbiol. 2014;52:3303-9.

32. Hofmann N, Mwingira F, Shekalaghe S, Robinson LJ, Mueller I, Felger I. Ultrasensitive detection of Plasmodium falciparum by amplification of multi-copy subtelomeric targets. PLoS Med. 2015;12:e1001788.

33. Kawamoto F, Miyake H, Kaneko O, Kimura M, Dung NT, Liu Q, et al. Sequence variation in the 18S rRNA genes, a target for PCR based malaria diagnosis, in Plasmodium ovale from southern Vietnam. J Clin Microbiol. 1996;34:2287e9.

34. Nguyen $\mathrm{HV}$, van den Eede $\mathrm{P}$, van Overmeir $\mathrm{C}$, Thang ND, le Hung $X$, D'Alessandro $U$, et al. Marked age-dependent prevalence of symptomatic and patent infections and complexity of distribution of human Plasmodium species in central Vietnam. Am J Trop Med Hyg. 2012;87:989-95.

35. Cox-Singh J. Knowlesi malaria in Vietnam. Malar J. 2009:8:269.

36. Pongvongsa T, Culleton R, Ha H, Thanh L, Phongmany P, Marchand RP, et al. Human infection with Plasmodium knowlesi on the Laos-Vietnam border. Trop Med Health. 2018;18(46):33.

37. Sullivan D. Uncertainty in mapping malaria epidemiology: implications for control. Epidemiol Rev. 2010;32:175-87.

38. Tirrell AR, Vendrely KM, Checkley LA, Davis SZ, McDew-White M, Cheese-

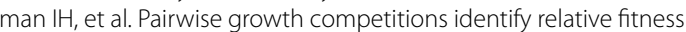
relationships among artemisinin resistant Plasmodium falciparum field isolates. Malar J. 2019:18:295.

39. Rogers WO, Sem R, Tero T, Chim P, Lim P, Muth S, et al. Failure of artesunate-mefloquine combination therapy for uncomplicated Plasmodium falciparum malaria in southern Cambodia. Malar J. 2009;8:10.

40. Spring MD, Pichyangkul S, Lon C, Gosi P, Yongvanichit K, Srichairatanakul $\mathrm{U}$, et al. Antibody profiles to plasmodium merozoite surface protein-1 in Cambodian adults during an active surveillance cohort with nested treatment study. Malar J. 2016;15:17

41. Helb DA, Tetteh KK, Felgner PL, Skinner J, Hubbard A, Arinaitwe E, et al. Novel serologic biomarkers provide accurate estimates of recent Plasmodium falciparum exposure for individuals and communities. Proc Natl Acad Sci USA. 2015:112:E4438-47. 
42. Kerkhof K, Sluydts V, Willen L, Kim S, Canier L, Heng S, et al. Serological markers to measure recent changes in malaria at population level in Cambodia. Malar J. 2016;15:529.

43. Longley RJ, França CT, White MT, Kumpitak C, Sa-angchai P, Gruszczyk J, et al. Asymptomatic Plasmodium vivax infections induce robust lgG responses to multiple blood-stage proteins in a low-transmission region of western Thailand. Malar J. 2017;16:178.

\section{Publisher's Note}

Springer Nature remains neutral with regard to jurisdictional claims in published maps and institutional affiliations.

- fast, convenient online submission

- thorough peer review by experienced researchers in your field

- rapid publication on acceptance

- support for research data, including large and complex data types

- gold Open Access which fosters wider collaboration and increased citations

- maximum visibility for your research: over $100 \mathrm{M}$ website views per year

At $\mathrm{BMC}$, research is always in progress.

Learn more biomedcentral.com/submissions 